\title{
Semiclassical Density Matrix Near the Top of a Potential Barrier
}

\author{
Franz Josef Weiper, Joachim Ankerhold and Hermann Grabert \\ Fakultät für Physik, Albert-Ludwigs-Universität Freiburg, \\ Hermann-Herder-Straße 4, 79104 Freiburg, Germany
}

\begin{abstract}
Employing the path integral approach, we calculate the semiclassical equilibrium density matrix of a particle moving in a nonlinear potential field for coordinates near the top of a potential barrier. As the temperature is decreased, near a critical temperature $T_{c}$ the harmonic approximation for the fluctuation path integral fails. This is due to a caustic arising at a bifurcation point of the classical paths. We provide a selfconsistent scheme to treat the large quantum fluctuations leading to a nonlinear fluctuation potential. The procedure differs from methods used near caustics of the real time propagator. The semiclassical density matrix is determined explicitly for the case of asymmetric barriers from high temperatures down to temperatures somewhat below $T_{c}$.
\end{abstract}

\section{Introduction}

Processes hindered by potential barriers play a central role in many areas of physics and chemistry [1]. When studying these systems, as a first step one has to determine the static behavior, that is the equilibrium density matrix $\rho_{\beta} \propto \exp (-\beta H)$. In barrier penetration problems the barrier height of the system is often large compared to the quantum mechanical energy level spacing. Then, a semiclassical evaluation of the equilibrium density matrix is justified. A consistent scheme to determine the coordinate representation of $\rho_{\beta}$ in the semiclassical approximation is provided by the path integral approach [2 [4]. Formally, the equilibrium density matrix may be viewed as a propagator in imaginary time. Hence, one first has to investigate the classical mechanics in the inverted nonlinear potential $-V(x)$. Afterwords, the quantum fluctuations about the classical paths are determined. Since the classical equation of motion, following from Hamilton's principle of least action, has to be solved with fixed endpoints, one finds in general a set of classical paths contributing to the path integral. If the classical paths, i.e. the minima of the classical action, 
are well separated in function space, the contribution of the quantum fluctuations around each path is given by the simple semiclassical approximation where the fluctuation path integral is evaluated in the Gaussian approximation. However, specific divergences, known as the problem of caustics, arise near those points where new classical paths become available. For a system with a potential barrier one encounters a caustic when the temperature is lowered, specifically in the temperature range where thermally activated barrier crossing changes over to quantum tunneling [5]. In this region one has to go beyond the Gaussian approximation for the fluctuation path integral. For the case of a symmetric potential field, the semiclassical approximation near the caustic was investigated in a previous paper [6]. Here, we extend the results to asymmetric barriers.

The paper is organized as follows. In section 2 we give a brief introduction to the path integral representation of the density matrix and the semiclassical approximation. In section 3 we then determine the classical paths and the classical action of a particle moving in the inverted barrier potential with endpoints in the vicinity of the barrier top. In section 4 we study the quantum fluctuations and determine the semiclassical density matrix at high temperatures and for temperatures near the caustic. Finally, in section 5 we give some explicit results for a barrier potential with cubic and quartic anharmonicities and present our conclusions.

\section{Path Integral Representation of the Density Matrix and Semiclassical Expansion}

The coordinate representation of the unnormalized equilibrium density matrix of a particle of mass $m$ moving in a potential $V(x)$ may be written as [2 [4]

$$
\rho_{\beta}\left(x, x^{\prime}\right)=\int \mathcal{D}[x] \mathrm{e}^{-\frac{1}{\hbar} S_{E}[x]}
$$

where the functional integral is over all paths $x(\tau), 0 \leq \tau \leq \hbar \beta$ with $x(0)=x$, $x(\hbar \beta)=x^{\prime}$. Each path is weighted by its Euclidian action

$$
S_{E}[x]=\int_{0}^{\hbar \beta} d \tau\left[\frac{1}{2} m \dot{x}^{2}+V(x)\right] .
$$

Since the density matrix can be regarded as the analytic continuation of the real time propagator to imaginary times $t \rightarrow-i \hbar \beta$, the representation (11) is frequently called the imaginary time path integral. The Euclidian action (2) describes the real time motion of a particle in the inverted potential $-V(x)$. 
Evaluation of the path integral (1) in an asymptotic expansion for $\hbar \rightarrow 0$ provides a consistent scheme for a semiclassical approximation. Thereby one first determines the maximum of the weighting factor, that is the minimum of $S_{E}[x]$. This is given by the classical action $S_{E}\left[x_{\mathrm{cl}}\right]$, where $x_{\mathrm{cl}}$ is the classical path solving the classical equation of motion following from Hamilton's principle $\delta S_{E}[x]=0$. An arbitrary path in (1) reads

$$
x(\tau)=x_{\mathrm{cl}}(\tau)+y(\tau)
$$

where $y(\tau)$ describes the quantum fluctuations about the classical path. The fluctuations have to fulfill the boundary conditions $y(0)=y(\hbar \beta)=0$. Using (3) the full action is then expanded around its minimum according to

$$
S_{E}[x]=S_{E}\left[x_{\mathrm{cl}}\right]+\sum_{n=2}^{\infty} \frac{1}{n !} \delta^{n} S_{E}\left[x_{\mathrm{cl}}, y\right]
$$

with the Frêchet derivatives

$$
\delta^{n} S_{E}\left[x_{\mathrm{cl}}, y\right]=\left.\int_{0}^{\hbar \beta} d \tau_{1} \cdots d \tau_{n} \frac{\delta^{n} S_{E}[x]}{\delta x\left(\tau_{1}\right) \cdots \delta x\left(\tau_{n}\right)}\right|_{x=x_{\mathrm{cl}}} y\left(\tau_{1}\right) \cdots y\left(\tau_{n}\right) .
$$

This way the dominant term (for $\hbar \rightarrow 0$ ) is separated off and one is left with a functional integral over closed paths. In the simple semiclassical approximation, the sum is truncated after the quadratic term leading to an exactly solvable Gaussian path integral [2 14. If there exists a set $\left\{x_{\mathrm{cl}}^{\alpha}\right\}$ of classical trajectories in $V(x)$, the above procedure must be performed for each $x_{\mathrm{cl}}^{\alpha}$, and all contributions are summed to yield the semiclassical density matrix

$$
\rho_{\beta}\left(x, x^{\prime}\right)=\sum_{\alpha} \frac{1}{\sqrt{J_{\alpha}}} \mathrm{e}^{-\frac{1}{\hbar} S_{E}\left[x_{\mathrm{cl}}^{\alpha}\right]}
$$

where $J_{\alpha}=\operatorname{det}\left\{\delta^{2} S_{E}[x] /\left.\delta x\left(\tau_{1}\right) \delta x\left(\tau_{2}\right)\right|_{x=x_{\mathrm{cl}}^{\alpha}}\right\}$ is the determinant describing the Gaussian integral over the quantum fluctuations [3, 14. Clearly, the result in (6) is exact if $V(x)$ is harmonic. $J_{\alpha}$ is given by the product of the eigenvalues $\Lambda_{n}^{\alpha}$ of the second order variational operator $\delta^{2} S_{E}[x] /\left.\delta x\left(\tau_{1}\right) \delta x\left(\tau_{2}\right)\right|_{x=x_{\mathrm{cl}}^{\alpha}}$ as

$$
J_{\alpha}=N \frac{2 \pi \hbar^{2} \beta}{m} \prod_{n} \Lambda_{n}^{\alpha}
$$

where $N$ is an appropriate normalization constant. As long as the second order variational operator is positive definite, i.e. $\Lambda_{n}>0$ for all $n$, the Gaussian 
approximation gives the leading order fluctuation term for $\hbar \rightarrow 0$. But a problem arises if one of the eigenvalues $\Lambda_{n}$ tends to zero, e.g. as the temperature is lowered. Then, the quantum fluctuations of this mode become arbitrarily large and the simple semiclassical approximation breaks down. Generally, the vanishing of an eigenvalue $\Lambda_{n}$ defines a point where new minimal action paths in the potential $V(x)$ become possible. This is well-known as the problem of caustics. In those regions where a caustic arises one has to go beyond the simple semiclassical approximation and has to consider higher order terms in the expansion (平) of the action.

\section{Classical Mechanics near the Barrier Top}

To determine the density matrix in the semiclassical approximation we first have to study the classical mechanics of the system in the inverted potential. Hence, we have to solve the classical equation of motion with the boundary conditions $x(0)=x, x(\hbar \beta)=x^{\prime}$, where the endpoints $x$ and $x^{\prime}$ are in the barrier region. A general barrier potential may be written as

$$
V(x)=-\frac{1}{2} M \omega^{2} x^{2}\left[1-2 \sum_{n=3}^{\infty} \frac{a_{n}}{n}\left(x / x_{a}\right)^{n-2}\right],
$$

where the $a_{n}$ are dimensionless coefficients that are of order 1 or smaller and $x_{a}$ is the characteristic distance from the barrier top at which anharmonic terms of the potential become relevant. We assume that for coordinates in the vicinity of the barrier top the potential (8) is only weakly affected by anharmonicities. This means that the length scale $x_{a}$ is much larger than the quantum mechanical length scale

$$
x_{0}=\left(\frac{\hbar}{2 M \omega}\right)^{1 / 2}
$$

which is the variance of the ground state of an harmonic oscillator in the inverted potential. Correspondingly,

$$
\epsilon=\frac{x_{0}}{x_{a}}
$$

is a small dimensionless parameter which will be serve as an expansion parameter for the semiclassical approximation in the following. This investigation becomes more transparent if we introduce the dimensionless coordinate

$$
q=x / x_{0}
$$


and the dimensionless time and inverse temperature

$$
\begin{aligned}
& \sigma=\omega \tau, \\
& \theta=\omega \hbar \beta .
\end{aligned}
$$

In terms of these dimensionless variables the scaled action $S=S_{E} / \hbar$ reads

$$
S\left[q, q^{\prime}\right]=\frac{1}{4} \int_{0}^{\theta} \mathrm{d} \sigma\left[\dot{q}^{2}(\sigma)-q^{2}(\sigma)\left(1-2 \sum_{n=3}^{\infty} \frac{a_{n}}{n} \epsilon^{n-2} q^{n-2}(\sigma)\right)\right] .
$$

To determine the classical paths in the time interval $0 \leq \sigma \leq \theta$, it is convenient to use the Fourier series expansion

$$
q(\sigma)=\frac{1}{\theta} \sum_{k=1}^{\infty} Q_{k} \sin \left(\nu_{k} \sigma\right)
$$

with the frequencies

$$
\nu_{k}=\frac{\pi k}{\theta} .
$$

The above series continues the path outside $[0, \theta]$ as an antisymmetric and periodic path with period $2 \theta$. The continued path has jump singularities at the endpoints of the interval $[0, \theta]$. This artefact of the Fourier series expansion must be taken into account when calculating time derivatives of $q$. Hence, in (13) one has to insert $\dot{q}_{\text {reg }}$ instead of $\dot{q}$ where

$$
\dot{q}_{\mathrm{reg}}(\sigma)=\dot{q}(\sigma)-c_{1}: \delta(\sigma, 2 \theta):-c_{2}: \delta(\sigma+\theta, 2 \theta): .
$$

Here, the coefficients $c_{1}, c_{2}$ are determined by the jumps at $2 k \theta$ and $(2 k-1) \theta$ as

$$
\begin{aligned}
& c_{1}=q\left(0^{+}\right)-q\left(0^{-}\right)=2 q \\
& c_{2}=q\left(\theta^{+}\right)-q\left(\theta^{-}\right)=-2 q^{\prime}
\end{aligned}
$$

and

$$
: \delta(\sigma, p):=\sum_{k=-\infty}^{\infty} \delta(\sigma-k p)
$$

is a periodically continued $\delta$-function. Inserting (14) and (16) into (13), the action is found to read in terms of the Fourier amplitudes 


$$
\begin{aligned}
S\left(q, q^{\prime}\right)= & \frac{1}{4 \theta}\left[\left(q-q^{\prime}\right)^{2}\right. \\
& +\frac{1}{2} \sum_{k=1}^{\infty}\left(\lambda_{k} Q_{k}^{2}-2 b_{k}\left(q, q^{\prime}\right) Q_{k}+4\left[q-(-1)^{k} q^{\prime}\right]^{2}\right) \\
& \left.+\sum_{n=3}^{\infty} \frac{a_{n}}{n}\left(\frac{\epsilon}{\theta}\right)^{n-2} \sum_{k_{1} \ldots k_{n}=1}^{\infty} D_{k_{1} \ldots k_{n}} Q_{k_{1}} \cdots Q_{k_{n}}\right]
\end{aligned}
$$

where we have introduced the coefficients

$$
D_{k_{1} k_{2} \cdots k_{n}}=2 \int_{0}^{1} \mathrm{~d} x \prod_{j=1}^{n} \sin \left(\pi k_{j} x\right)
$$

and

$$
\lambda_{k}=\left(\frac{\pi k}{\theta}\right)^{2}-1
$$

as well as

$$
b_{k}\left(q, q^{\prime}\right)=\frac{2 \pi k}{\theta}\left[q-(-1)^{k} q^{\prime}\right]
$$

Requiring that the variation $\delta S\left[Q_{k}\right]=0$, we get the Fourier representation of the classical equation of motion

$$
\lambda_{k} Q_{k}=b_{k}-\sum_{n=3}^{\infty} a_{n}\left(\frac{\epsilon}{\theta}\right)^{n-2} \sum_{k_{1}, \cdots, k_{n-1}=1}^{\infty} D_{k_{1} k_{2} \cdots k_{n-1} k} Q_{k_{1}} \cdots Q_{k_{n-1}}
$$

In general, the nonlinear equations (23) cannot be solved exactly. However, for small $\epsilon$ and not too large $\theta$ one may solve them pertubatively in a selfconsistent manner.

\subsection{High Temperatures}

Since the boundary values $q, q^{\prime}$ are in the barrier region and assumed to be of order 1 or smaller, for high temperatures, $\theta \ll 1$, the $b_{k}\left(q, q^{\prime}\right)$ are of order $1 / \theta$ or smaller. The anharmonic terms in (23) are then at most of order $\epsilon \theta$ and the classical equation of motion reduces to

$$
\lambda_{k} Q_{k}=b_{k}+\mathcal{O}(\epsilon \theta) .
$$


Neglecting the corrections, we obtain the Fourier representation of the minimal action paths of an harmonic oscillator. When (24) is inserted into (19), the corresponding action is found to read

$$
S(z, r)=-\frac{r^{2}}{2} \tan \left(\frac{\theta}{2}\right)+\frac{z^{2}}{8} \cot \left(\frac{\theta}{2}\right)+\mathcal{O}\left(\epsilon \theta^{2}\right),
$$

where we have introduced dimensionless sum and difference coordinates

$$
r=\frac{q+q^{\prime}}{2}, z=q-q^{\prime}
$$

This approximation is only valid for endpoints within the barrier region and temperatures where the amplitude of the classical path remains within this domain. Clearly, the harmonic approximation fails if one of the coefficients $\lambda_{k}$ vanishes. When the temperature is lowered $\lambda_{1}$ reaches zero at the critical temperature

$$
T_{c}=\frac{\hbar \omega}{\pi k_{\mathrm{B}}}
$$

i.e. $\theta_{c}=\pi$, and the corresponding amplitude $Q_{1}$ diverges. Corresponding singularities of the harmonic approximation arise for all temperatures where one of the frequencies $\nu_{k}=1$. This is not surprising, rather it reflects the typical behavior of a harmonic oscillator in real time. For a harmonic potential the oscillation period $2 \pi$ is independent of the amplitude of the path. Hence, for times $\theta=n \pi$, corresponding to multiples of half the oscillation period, there are infinitely many paths with arbitrary large amplitudes connecting $q$ and $q^{\prime}=(-1)^{n} q$.

\subsection{Classical Paths Near $T_{c}$}

When the temperature approaches $T_{c}$ from above, the eigenvalue $\lambda_{1}$ tends to zero and the harmonic approximation fails. Then, the anharmonic terms in (23) become important. In general, the equation of motion cannot be solved analytically near $T_{c}$. However, the classical paths and their actions can always be computed numerically. Here, we consider suitable potentials (see below) where only the amplitude $Q_{1}$ increases. For the $b_{k}\left(q, q^{\prime}\right)$ are now of order one or smaller and for perturbatively small $\epsilon$ we shall assume that for temperatures near $T_{c}$ the amplitude $Q_{1}$ may become at most of order $\epsilon^{-2 / 3}$, while all other amplitudes are of order 1 or smaller. This order of magnitude of the

Fourier coefficients will be confirmed below for appropriate barrier shape. The 


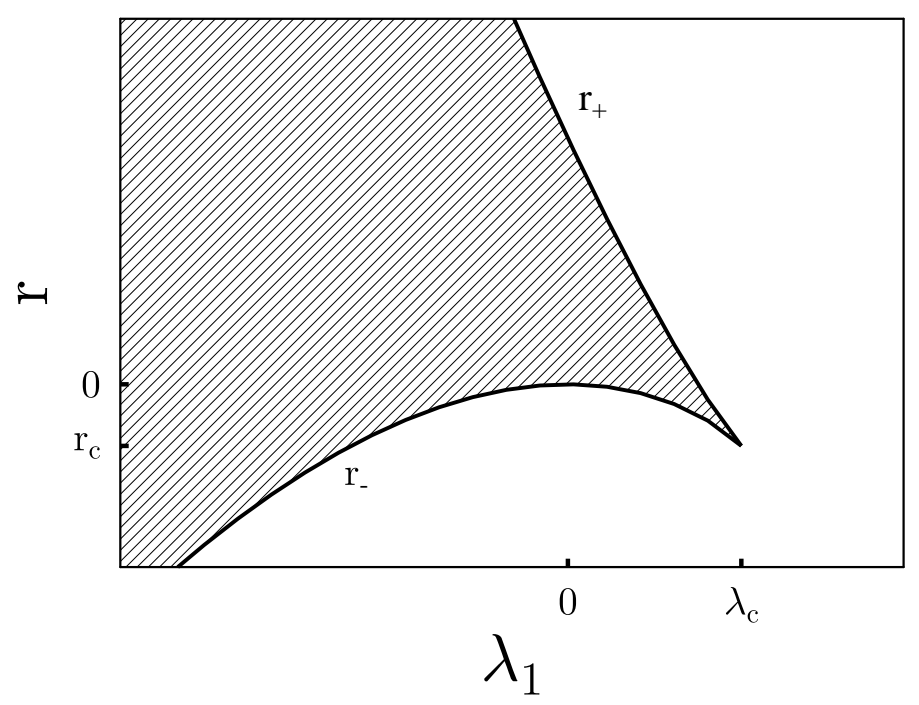

Fig. 1. The $\lambda_{1}-r$-plane is divided by the two curves $r_{ \pm}\left(\lambda_{1}\right)$ (solid lines) into two regions in which the cubic equation (30) has one or three (shaded region) solutions. equation of motion (23) then reduces to

$$
\lambda_{k} Q_{k}=b_{k}-a_{3} \frac{\epsilon}{\theta} D_{11 k} Q_{1}^{2}-a_{4}\left(\frac{\epsilon}{\theta}\right)^{2} D_{111 k} Q_{1}^{3}+\mathcal{O}\left(\epsilon^{2 / 3}\right) .
$$

Within this approximation the amplitudes $Q_{n}$ for $n>1$ are coupled to $Q_{1}$ only. We have to solve a cubic equation for the mode amplitude $Q_{1}$ and linear equations for the other mode amplitudes $Q_{n}$. To derive (28) we have made two assumptions. First, the parameter $a_{4}$ is assumed to be positive and of order 1. In fact, the lenghtscale $x_{a}$ in (8) can always be chosen such that $a_{4}=1$ unless $a_{4}$ is not positive. Second, the coefficient $a_{3}$ can be taken positive without loss of generality. The coefficients $a_{2 n+1}, n \geq 1$ are assumed to be smaller than $\epsilon^{1 / 3}$ which means that the barrier potential should be only weakly asymmetric. Only then, the cubic equation (28) has real solutions $Q_{1}$ at most of order $\epsilon^{-2 / 3}$ for all endpoints in the barrier region as assumed in deriving (28) and correction terms of order $\epsilon^{2 / 3}$. For larger asymmetries global features of the potential become relevant already in the vicinity of $T_{c}$. To make the $\epsilon$-dependence more transparent we set

$$
Q=\frac{1}{2 \theta} \epsilon^{2 / 3} Q_{1}
$$

Then, using $D_{111}=8 / 3 \pi, D_{1111}=3 / 4$, and $b_{1}=4 \pi r / \theta$, the cubic equation for the mode amplitude $Q$ takes the form

$$
3 a_{4} Q^{3}+\frac{16}{3 \pi} a_{3} \epsilon^{-1 / 3} Q^{2}+\lambda_{1} \epsilon^{-2 / 3} Q=\frac{2 \pi}{\theta^{2}} r .
$$




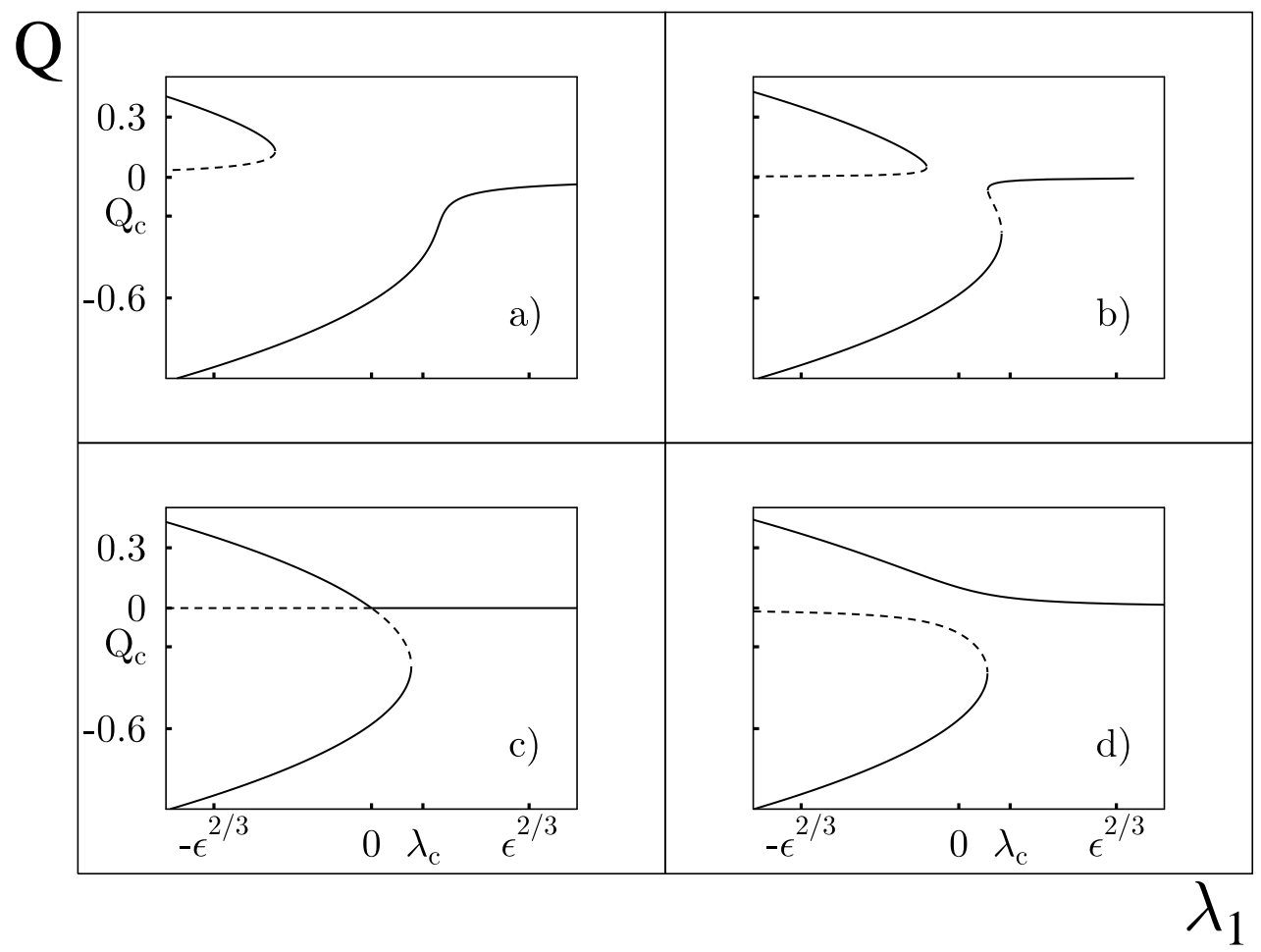

Fig. 2. Bifurcation scenario for the classical paths for various boundary values $r$, a) $r<r_{c}$, b) $r_{c}<r<0$, c) $r=0$, d) $r>0$. The solid (dashed) lines represent the stable (unstable) solutions of (30). The potential parameter are $a_{3}=1 / 5, a_{4}=1$ and $\epsilon=0.01$.

From a mathematical point of view (30) describes a pure $(r=0)$ or a perturbed $(r \neq 0)$ one-dimensional bifurcation problem with bifurcation parameter $\lambda_{1}$ [8]. The cubic equation (30) implies two curves

$$
r_{ \pm}\left(\lambda_{1}\right)=r_{c}\left[3 \frac{\lambda_{1}}{\lambda_{c}}-2 \pm 2\left(1-\frac{\lambda_{1}}{\lambda_{c}}\right)^{3 / 2}\right]
$$

in the $\lambda_{1}-r$-plane where bifurcations occur (Fig. 1). Here, the critical point $\left(\lambda_{c}, r_{c}\right)$ is given by

$$
\begin{aligned}
& \lambda_{c}=9 a_{4} Q_{c}^{2} \epsilon^{2 / 3} \\
& r_{c}=\frac{3 a_{4} \theta^{2}}{2 \pi} Q_{c}^{3},
\end{aligned}
$$

where

$$
Q_{c}=-\frac{16 a_{3}}{27 \pi a_{4}} \epsilon^{-1 / 3}
$$


is the corresponding amplitude. Note that $\lambda_{c}=(4 / 3)^{4} a_{3}^{2} / a_{4} \pi^{2}$ is independent of $\epsilon$. Further, $r_{c}<0$, and $Q_{c}$ is of order one or smaller for $a_{3}, a_{4}>0$ as assumed above.

For $\lambda_{1}>\lambda_{c}$ there is only one solution $Q$ of (30) which has the same sign as $r$. As the temperature is lowered, i.e. $\lambda_{1}$ is decreased, several cases must be distinguished. First, for $r<r_{c}$ the high temperature solution continuously extents to the region $\lambda_{1}<\lambda_{c}$ and the amplitude $|Q|$ grows (Fig. Za). Two new solutions with positive sign emerge when the temperature reaches the point where $r_{-}\left(\lambda_{1}\right)=r$ (Fig. 1). One of the new solutions is stable and the other unstable. The amplitude of the stable (unstable) path increases (decreases) with decreasing $\lambda_{1}$. Second, for $r_{c} \leq r<0$ one first reaches a point where $r_{+}\left(\lambda_{1}\right)=r$ when the temperature is decreased (Fig. 1). At this temperature two new branches with negative sign but larger amplitude $|Q|$ appear (Fig. 20/b). Again one path is stable and the other is unstable. With decreasing $\lambda_{1}$ the amplitude $|Q|$ of the unstable path decreases and approaches the stable solution which extends from the high temperature region. These two paths vanish when the temperature reaches the value where $r_{-}\left(\lambda_{1}\right)=r$. For temperatures below this point there exist only one stable solution until we arrive at the second solution of $r_{-}\left(\lambda_{1}\right)=r$. Here a stable and unstable path with positive sign emerge where the amplitude of the stable (unstable) path increases (decreases) as the temperature is lowered further. For $r=0$ (Fig. 2c) we have a double point bifurcation scenario described below. Finally, for $r>0$ (Fig. 2d) two new paths appear at the point where $r_{+}\left(\lambda_{1}\right)=r$. Again one solution is stable and the other one is unstable.

In particular, for $r=0$ the solutions of (30) are (Fig. 2 $\mathrm{c}$ )

$$
Q=\left\{\begin{array}{l}
0 \\
Q_{0}\left(1-\sqrt{1-\frac{\lambda_{1}}{\lambda_{0}}}\right) \\
Q_{0}\left(1+\sqrt{1-\frac{\lambda_{1}}{\lambda_{0}}}\right)
\end{array}\right.
$$

where $Q_{0}$ and $\lambda_{0}$ are given by

$$
\begin{aligned}
Q_{0} & =\frac{3}{2} Q_{c} \\
\lambda_{0} & =\frac{3}{4} \lambda_{c}
\end{aligned}
$$

with $\lambda_{c}$ and $Q_{c}$ from (32) and (33), respectively. One can show, and it will emerge below, that the trivial solution is stable for $\lambda_{1} \geq 0$, the second solution is stable for $\lambda_{1} \leq \lambda_{0}$, while the third solution is stable for $\lambda_{1} \leq 0$. 


\subsection{Classical Action Near $T_{c}$}

Having determined the classical path we are able to calculate the minimal action. Since (30) may easily be solved numerically, the action will be given as a function of the amplitude $Q$ of the large amplitude mode. To get explicit

values for the action the numerical value for $Q$ must be inserted. Now, inserting (14) with the Fourier amplitudes determined from (28) and (30) into (19) and expanding in powers of $\epsilon$, we obtain after some algebra

$$
\begin{aligned}
S_{\mathrm{cl}}(Q, z, r)= & \frac{r^{2}}{\theta} \Lambda+\frac{z^{2}}{\theta} \Omega-\frac{\pi r}{\theta} \epsilon^{-2 / 3} Q-\left(\frac{8 a_{3} \theta}{9 \pi \epsilon^{1 / 3}} Q^{3}+\frac{3 a_{4} \theta}{4} Q^{4}\right) \epsilon^{-2 / 3} \\
& -\frac{4 a_{3} \theta(2+3 \Lambda) r}{3\left(4 \pi^{2}-\theta^{2}\right) \epsilon^{1 / 3}} Q^{2}-\frac{12 \pi a_{4} r}{2 \theta \lambda_{3}} Q^{3}-\frac{2 a_{3}^{2} \theta \Gamma}{\epsilon^{2 / 3}} Q^{4} \\
& +\frac{2 a_{3} a_{4} \theta}{\epsilon^{1 / 3} \lambda_{3}} Q^{5}-\frac{a_{4}^{2} \theta}{2 \lambda_{3}} Q^{6}+\frac{256 a_{5} \theta}{75 \pi \epsilon^{1 / 3}} Q^{5}+\frac{5 a_{6} \theta}{3} Q^{6}+\mathcal{O}\left(\epsilon^{2 / 3}\right)(36)
\end{aligned}
$$

Here we have introduced the coefficients

$$
\begin{aligned}
& \Lambda=-\frac{\theta}{2} \tan \left(\frac{\theta}{2}\right)+\frac{2 \pi}{\pi^{2}-\theta^{2}} \\
& \Omega=\frac{\theta}{8} \cot \left(\frac{\theta}{2}\right) \\
& \Gamma=\frac{3}{4}-\frac{\pi^{2}}{4 \pi^{2}-\theta^{2}}+\left(\frac{4 \pi^{2}}{4 \pi^{2}-\theta^{2}}\right)^{2} \frac{1}{\theta} \tan \left(\frac{\theta}{2}\right)-\frac{64 \theta^{2}}{9 \pi^{2}\left(\pi^{2}-\theta^{2}\right)},
\end{aligned}
$$

which remain finite in the limit $\theta \rightarrow \pi$, i.e.

$$
\begin{aligned}
& \lim _{\theta \rightarrow \pi} \Lambda=\frac{3}{2} \\
& \lim _{\theta \rightarrow \pi} \Omega=0 \\
& \lim _{\theta \rightarrow \pi} \Gamma=\frac{5}{12}-\frac{16}{3 \pi^{2}} .
\end{aligned}
$$

The first three terms in (36) reduce to the usual harmonic action for temperatures well above $T_{c}$. Indeed, for $\lambda_{1}$ larger than order $\epsilon^{2 / 3}$ the anharmonic terms 
in (30) can be neglected and we have

$$
Q=\frac{2 \pi r}{\theta^{2} \lambda_{1}} \epsilon^{2 / 3}+\mathcal{O}\left(\frac{\epsilon^{2}}{\lambda_{1}^{3}}\right)
$$

and hence

$$
r^{2} \Lambda-\pi r \epsilon^{-2 / 3} Q=-\frac{\theta}{2} r^{2} \tan \left(\frac{\theta}{2}\right)\left[1+\mathcal{O}\left(\frac{\epsilon^{4 / 3}}{\lambda_{1}^{2}}\right)\right] .
$$

While the harmonic action diverges for $\theta \rightarrow \pi$, i.e. $\lambda_{1} \rightarrow 0$, the full action (36) remains finite due to the contributions of the anharmonic terms.

In the special case $q=q^{\prime}=0$, i.e. $r=x=0$, one sees that for high temperatures the minimal action vanishes. With decreasing $\lambda_{1}$ two new paths emerge at $\lambda_{1}=\lambda_{0}$. At the bifurcation point the amplitudes of the new paths coincide, and to leading order the action is given by

$$
S_{\mathrm{cl}}\left(Q_{0}, 0,0\right)_{\lambda_{1}=\lambda_{0}}=\frac{\theta}{4} a_{4} Q_{0}^{4} \epsilon^{-2 / 3}+\mathcal{O}(1) .
$$

Since $S_{\mathrm{cl}}\left(Q_{0}, 0,0\right)_{\lambda_{1}=\lambda_{0}}>0$, the trivial solution $(Q=0$ branch $)$ is absolutely stable. At lower temperatures, i.e. smaller $\lambda_{1}$, the solutions separate. Denoting the stable and unstable branch by $Q_{s}$ and $Q_{u}$, respectively, the new amplitudes reach $Q_{s}=2 Q_{0}$ and $Q_{u}=0$, at $\lambda_{1}=0$, and the corresponding actions are given by

$$
\begin{aligned}
& S_{\mathrm{cl}}\left(Q_{s}, 0,0\right)_{\lambda_{1}=0}=-4 \theta a_{4} Q_{0}^{4} \epsilon^{-2 / 3}+\mathcal{O}(1) \\
& S_{\mathrm{cl}}\left(Q_{u}, 0,0\right)_{\lambda_{1}=0}=0 .
\end{aligned}
$$

Now, $S_{\mathrm{cl}}\left(Q_{s}, 0,0\right)_{\lambda_{1}=\lambda_{0}}<0$, and the $Q_{s}$ branch is absolutely stable. Between $\lambda_{1}=\lambda_{0}$ and $\lambda_{1}=0$ at the point $\lambda_{1}=2 \lambda_{c} / 3$ the two stable paths have to leading order the same action and they exchange global stability.

This example demonstrates the change of stability of the classical paths. At high temperatures there is only one minimum of the action in function space. With decreasing temperature a saddlepoint emerges at $\lambda_{1}=\lambda_{0}$ with an action larger than the minimal action. This saddlepoint splits into a maximum and a second minimum of the action. The original minimum and the new minimum exchange global stability at $\lambda_{1}=2 \lambda_{c} / 3$. Finally, the original minimum and the maximum meet in a double point at $\lambda_{1}=0$.

For finite $q$ and $q^{\prime}$ the cubic equation (30) may easily be solved numerically. Again new extrema of the action functional arise as the temperature is lowered. 
However, a change of global stability only occurs for small values of $r$. A more detailed discussion follows in section 4.4.

\section{Quantum Fluctuations}

Based on the classical paths and the associated extremal actions, we proceed to determine the equilibrium density matrix by expanding the action about the classical paths according to (田).

We split an arbitrary path $q(\sigma)$ into the classical path $q_{\mathrm{cl}}(\sigma)$ and a fluctuation $y(\sigma)$. The relevant fluctuations give a contribution to the full action of order $\hbar$. Therefore, the semiclassical expansion is only consistent if the classical action is also determined at least to order $\hbar$ that is to order 1 in the dimensionless units used above. In (36) we have neglected contributions to the classical action that are smaller than 1 for coordinates of order 1 . On the basis of this result we may determine the semiclassical density matrix in the vicinity of the barrier top. For coordinates that are larger than order 1 a semiclassical expansion is of course still feasible, but mostly one has to use numerical methods to go beyond the approximate results derived in the previous section. The exception are some special potentials, for instance the Eckart barrier potential, where the classical mechanics can be solved exactly [7].

\subsection{Expansion about the Classical Path}

To evaluate the pathintegral an arbitrary path is decomposed into

$$
q(\sigma)=q_{\mathrm{cl}}(\sigma)+y(\sigma)
$$

and one has to expand the action about $q_{\mathrm{cl}}$. If there exist a set $q_{\mathrm{cl}}^{\alpha}(\sigma)$ of stable classical paths, the action is expanded about each $q_{\mathrm{cl}}^{\alpha}(\sigma)$. Then the density matrix (囵) may be written as

$$
\rho_{\theta}(z, r)=\frac{1}{Z} \sum_{\alpha} \exp \left(-S_{c l}\left[Q_{\alpha}, z, r\right]\right) f\left(Q_{\alpha}, z, r\right)
$$

where $S_{\mathrm{cl}}[Q, z, r]$ is the scaled minimal action (13) and $Z$ denotes an appropriate normalization constant. $f\left(Q_{\alpha}, z, r\right)$ is a functional integral over the paths $y(\sigma)$ given by

$$
f(Q, x, r)=\int \mathcal{D}[y] \exp \left(-\sum_{m=2}^{\infty} \delta^{m} S\left[q_{\mathrm{cl}}, y\right]\right),
$$


where $\delta^{m} S\left[q_{\mathrm{cl}}, y\right]$ are scaled Frêchet derivatives according to (5). Since the fluctuations $y(\sigma)$ have to satisfy the boundary conditions $y(0)=y(\theta)=0$, we use the Fourier representation

$$
y(\sigma)=\frac{1}{\theta} \sum_{k=1}^{\infty} Y_{k} \sin \left(\nu_{k} \sigma\right) \text {. }
$$

Then, the integration measure becomes

$$
\int \mathcal{D}[y] \cdots=\prod_{n=1}^{\infty}\left(\frac{1}{N} \sqrt{\frac{1}{8 \pi \theta}} \int_{-\infty}^{\infty} \mathrm{d} Y_{n} \cdots\right)
$$

where $N$ can be determined from the limit of a free particle, i.e. $\omega \rightarrow 0$. With the Fourier representations of the classical path (14) and the fluctuations (46), respectively, one finds for the scaled Frêchet derivatives

$$
\begin{aligned}
\delta^{2} S\left[q_{\mathrm{cl}}, y\right]= & \frac{1}{8 \theta} \sum_{k=1}^{\infty} \lambda_{k} Y_{k}^{2}+\frac{1}{8 \theta} \sum_{n=3}^{\infty} a_{n}(n-1)\left(\frac{\epsilon}{\theta}\right)^{n-2} \\
& \times \sum_{k_{1} \ldots k_{n}=1}^{\infty} D_{k_{1} \ldots k_{n}} Q_{k_{1}} \cdots Q_{k_{n-2}} Y_{k_{n-1}} Y_{k_{n}}
\end{aligned}
$$

and

$$
\begin{aligned}
\delta^{m} S\left[q_{\mathrm{cl}}, y\right]= & \frac{1}{4 \theta} \sum_{n=m}^{\infty} a_{n} \frac{(n-1) !}{m !(n-m) !}\left(\frac{\epsilon}{\theta}\right)^{n-2} \\
& \times \sum_{k_{1} \ldots k_{n}=1}^{\infty} D_{k_{1} \ldots k_{n}} Q_{k_{1}} \cdots Q_{k_{n-m}} Y_{k_{n-m+1}} \cdots Y_{k_{n}}
\end{aligned}
$$

for $m \geq 3$. Here, the coefficients $\lambda_{k}$ and $D_{k_{1} \ldots k_{n}}$ are defined in (21) and (20), respectively. The first term of (48) arises from the bilinear terms of the action, while the remaining terms are due to the anharmonicity of the potential. Clearly, from (49) we see that the semiclassical expansion proceeds in powers of $\epsilon$. Of course, the fluctuation integral (45) cannot be done exactly. However, for small $\epsilon$ we may evaluate the functional integral (45) perturbatively. To do so, we first have to solve (23) perturbatively for small $\epsilon$. In particular, this yields the order of magnitude of the Fourier coefficients $Q_{k}$ that depends on the temperature range. Afterwards, one estimates the size of the Fourier coefficients $Y_{k}$ of the relevant fluctuations. The exponent of the integrand of (45), given by (48) and (49), is an expansion in powers of $Y_{k}$ with coefficients depending on the parameter $\epsilon$, if we express the order of magnitude of the $Q_{k}$ in 
terms of $\epsilon$. In a first step the size of the $Y_{k}$ is estimated from the assumption that the terms in the action quadratic in $Y_{k}$ are of order 1. However, this estimate is only correct if the terms of higher order in $Y_{k}$ vanish in the limit $\epsilon \rightarrow 0$. If there are higher order terms that grow in this limit one gets a new (smaller) estimate of the $Y_{k}$ by requiring that the leading order stabilizing terms are of order 1. Again the estimate depends on the range of temperatures considered.

\subsection{High Temperatures}

Above, when studying the classical mechanics we have seen that for temperatures $\theta \ll \pi$ and for coordinates near the barrier top the anharmonic terms are neglectible. In the limit of small $\epsilon$ and for high temperatures the magnitude of the Fourier coefficients $Q_{k}$ is of order $\theta$. Assuming that the coefficients $Y_{k}$ are of order $\theta^{3 / 2}$ or smaller we see that the first term on the right hand side of (48) is of order 1 , while the remaining terms of (48) and (49) vanish in the limit $\epsilon \rightarrow 0$. Therefore, for the relevant fluctuations the exponent of (45) is given by

$$
S[q]-S\left[q_{c l}\right]=\frac{1}{8 \theta} \sum_{n=1}^{\infty} \lambda_{n} Y_{n}^{2}+\mathcal{O}\left(\epsilon \theta^{2}\right)
$$

Thus, the path integral (45) reduces to a product of independent Gaussian integrals

$$
f=\frac{1}{N} \prod_{k=1}^{\infty}\left(\sqrt{\frac{1}{8 \pi \theta}} \int_{-\infty}^{\infty} \mathrm{d} Y_{k} \exp \left(-\frac{1}{8 \theta} \lambda_{k} Y_{k}^{2}\right)\right)
$$

where the eigenvalues $\lambda_{k}$ of the second variational operator are given by (21). Now, the fluctuation prefactor for the equilibrium density matrix is obtained as

$$
f=[4 \pi \sin (\theta)]^{-1 / 2}
$$

where the normalization in (51) is derived from the free particle limit. Combining the action (25) and the fluctuation prefactor (52), the equilibrium density matrix for coordinates near the barrier top and for high temperatures emerges as

$$
\rho_{\theta}(z, r)=\frac{1}{Z} \sqrt{\frac{1}{4 \pi \sin (\theta)}} \exp \left[-\frac{r^{2}}{2} \tan \left(\frac{\theta}{2}\right)+\frac{z^{2}}{8} \cot \left(\frac{\theta}{2}\right)\right] .
$$


Hence, in this temperature and coordinate range, we regain the well known result for the inverted harmonic oscillator. Clearly for $\theta \rightarrow \pi$, this result becomes singular. Then, the mode $Y_{1}$ undergoes large fluctuations and one has to go beyond the simple semiclassical approximation.

\subsection{Quantum Fluctuations Near $T_{c}$}

Since the eigenvalue $\lambda_{1}$ of the second variational operator vanishes for $T=T_{c}$, the simple semiclassical approximation fails near $T_{c}$. Under the assumptions made in section 3.2 about the potential parameters $a_{n}$ we were able to solve the classical equation of motion (23) near the critical temperature perturbatively for small $\epsilon$. In this temperature range all amplitudes $Q_{k}$ for $k>1$ are of order 1 or smaller, while the magnitude of the amplitude $Q_{1}$ is of order $\epsilon^{-2 / 3}$ or smaller. We now estimate the magnitude of the Fourier coefficients $Y_{k}$. The amplitudes $Y_{k}$ for $k>1$ are of order 1 or smaller since all eigenvalues $\lambda_{k}$ for $k>1$ are of order 1 in this temperature range. Hence, the fluctuations $Y_{k}$ for $k>1$ are bounded by the first term on the right hand side of (48).

Near the critical temperature the amplitude $Y_{1}$ can become much larger than 1. To estimate the size of $Y_{1}$ we first consider the terms of (48) that are quadratic in $Y_{1}$. With (32) and (33) we have

$$
\begin{aligned}
\delta^{2} S\left[q_{\mathrm{cl}}, y\right]- & \frac{1}{8 \theta} \sum_{k=2}^{\infty} \lambda_{k} Y_{k}^{2}= \\
& \frac{1}{8 \theta}\left(\lambda_{1}-\lambda_{c}+9 a_{4} \epsilon^{2 / 3}\left(Q-Q_{c}\right)^{2}\right) Y_{1}^{2}\left[1+\mathcal{O}\left(\epsilon^{1 / 2}\right)\right] .
\end{aligned}
$$

From (54) we see that the coefficient

$$
\Lambda_{1}(Q)=\lambda_{1}-\lambda_{c}+9 a_{4} \epsilon^{2 / 3}\left(Q-Q_{c}\right)^{2}
$$

vanishes at the critical point, where $\lambda_{1}=\lambda_{c}$ and $Q=Q_{c}$. Thus the size of $Y_{1}$ is only bounded by higher order terms in $Y_{1}$. If we assume that $Y_{1}$ is of order $\epsilon^{-1 / 2}$ or smaller near the critical point, the fourth order variational term gives

$$
\delta^{4} S\left[q_{\mathrm{cl}}, y\right]=\frac{3 a_{4}}{64 \theta^{3}} \epsilon^{2} Y_{1}^{4}+o(1)
$$

Indeed, this term limits the size of the mode amplitude $Y_{1}$ near $\lambda_{1}=\lambda_{c}$ to the assumed order of magnitude. With this estimate for $Y_{1}$ and in a narrow range 
around the critical point, where $\Lambda_{1}(Q) \leq \mathcal{O}(\epsilon)$ one gets for the action of the fluctuations

$$
S[q]-S\left[q_{c l}\right]=\frac{1}{8 \theta} \sum_{n=2}^{\infty} \lambda_{n} Y_{n}^{2}+V\left(Q, Y_{1}\right)+\mathcal{O}\left(\epsilon^{1 / 2}\right),
$$

with the fluctuation potential

$$
\begin{aligned}
V\left(Q, Y_{1}\right)=\frac{1}{8 \theta}[( & \left.\lambda_{1}-\lambda_{c}+9 a_{4} \epsilon^{2 / 3}\left(Q-Q_{c}\right)^{2}\right) Y_{1}^{2} \\
& \left.+3 a_{4} \frac{\epsilon^{4 / 3}}{\theta}\left(Q-Q_{c}\right) Y_{1}^{3}+\frac{3 a_{4}}{8}\left(\frac{\epsilon}{\theta}\right)^{2} Y_{1}^{4}\right] .
\end{aligned}
$$

From (55) we see that the coefficient $\Lambda_{1}(Q)$ of the harmonic term may vanish. This is indeed the case for values of $r$ and $\lambda_{1}$ on the two curves $r^{ \pm}\left(\lambda_{1}\right)$ introduced in (31). When these curves are crossed, the classical equation of motion (30) has a bifurcation. Near the bifurcation the remaining terms of the fluctuation potential always constrain $Y_{1}$ to fluctuation amplitudes of or$\operatorname{der} \epsilon^{-1 / 2}$ as assumed above. For $\Lambda_{1}(Q)>0$ the fluctuation potential has only one minimum at $Y_{1}=0$, corresponding to one solution of the classical equation of motion. For $\Lambda_{1}(Q) \leq 0$ the fluctuation potential has three extrema, at $Y_{1}=0$ and at

$$
Y_{ \pm}=\frac{-3 \theta}{\epsilon^{2 / 3}}\left(Q-Q_{c}\right) \pm \sqrt{-\frac{4 \theta^{2}}{3 a_{4} \epsilon^{2}}\left(\lambda_{1}-\lambda_{c}\right)-\frac{3 \theta^{2}}{\epsilon^{4 / 3}}\left(Q-Q_{c}\right)^{2}}
$$

Specifically, let us discuss the fluctuation potential for fixed $Q=Q_{c}$. Hence, according to (30), we consider values along a line $r=r_{e}\left(\lambda_{1}\right)$ in the $\lambda_{1}$-r-plane, where

$$
r_{e}\left(\lambda_{1}\right)=r_{c}\left(3 \frac{\lambda_{1}}{\lambda_{c}}-2\right)
$$

For fixed $Q=Q_{c}$ the fluctuation potential has the simple form

$$
V\left(Q_{c}, Y_{1}\right)=\frac{1}{8 \theta}\left[\left(\lambda_{1}-\lambda_{c}\right) Y_{1}^{2}+\frac{3}{8} a_{4}\left(\frac{\epsilon}{\theta}\right)^{2} Y_{1}^{4}\right]
$$

which is symmetric about $Y_{1}=0$ (Fig. 3). According to (59) for $\lambda_{1}<\lambda_{c}$ the 


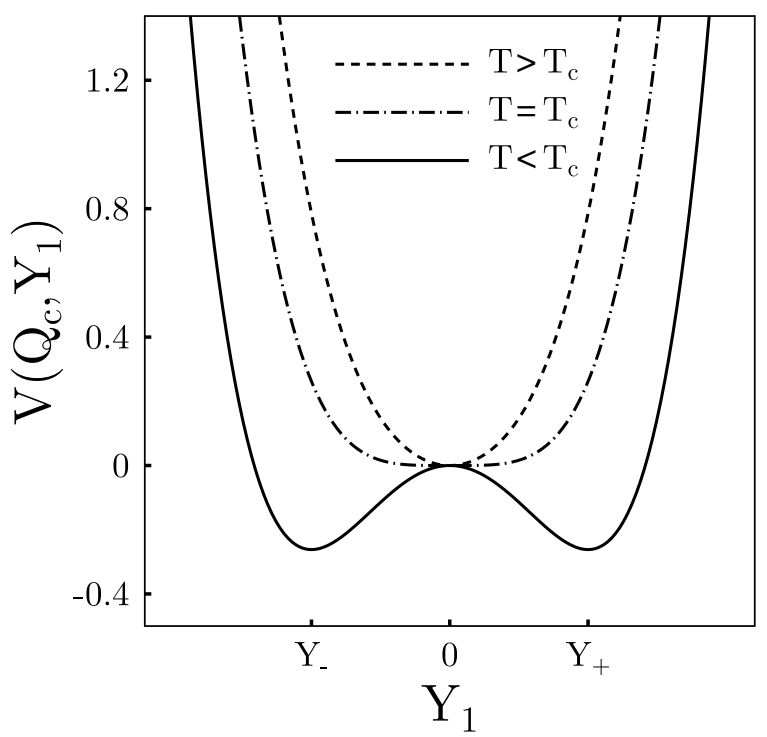

Fig. 3. The fluctuation potential (58) for $Q=Q_{c}$ and temperatures near $T_{c}$.

extrema $Y_{ \pm}$are at

$$
Y_{ \pm}= \pm \sqrt{\frac{4 \theta^{2}}{3 a_{4} \epsilon^{2}}\left(\lambda_{c}-\lambda_{1}\right)}
$$

For $\lambda_{1}-\lambda_{c}>\epsilon$ the fluctuation potential has only one minimum $Y_{1}=0$ and the second order variational operator constrains $Y_{1}$ to fluctuation amplitudes smaller than order $\epsilon^{-1 / 2}$. In this region the simple semiclassical approximation, i.e. the truncation after the quadratic term in the fluctuation potential, is valid. For $\lambda_{1}$ in the region $0<\lambda_{1}-\lambda_{c}<\epsilon$, the curvature of the fluctuation potential at the minimum at $Y_{1}=0$ becomes smaller and the fluctuation amplitude of the marginal mode is stabilized only by the quartic term. When $\lambda_{1}$ is decreased further, the minimum at $Y_{1}=0$ becomes a maximum and new minima arise at $Y_{ \pm}$according to the bifurcation scenario discussed for the classical paths. Indeed, one can show that the extrema of the fluctuation potential $V\left(Q, Y_{1}\right)$ are the solutions of the cubic equation (30) for $Q$ apart from the scaling factor $2 \theta \epsilon^{-2 / 3}$ introduced in (29). In the region $-\epsilon<\lambda_{1}-\lambda_{c}<0$ the new minima are not well separated by the local maximum at $Y_{1}=0$ and fluctuations from one minimum to the other occur. Hence, in this region the fluctuations are also stabilized by the quartic term in the fluctuation potential. When the temperature is lowered further the barrier height of the local maximum of the fluctuation potential becomes larger and the fluctuations about the minima decrease. Then the system is stabilized near the stable classical paths and a Gaussian approximation for the integral over the fluctuations around these paths is again appropriate. 


\subsection{The Density Matrix Near $T_{c}$}

Well above $T_{c}$ the density matrix is given by (53). As $T_{c}$ is approached the fluctuations grow, and one has to take into account the full fluctuation potential (58) which stabilizes the fluctuations by the quartic term. In the vicinity of the critical point $\left(\lambda_{c}, r_{c}\right)$ the various solutions of the cubic equation (30) are not well separated and the question arise, which branch must be inserted into the action and the fluctuation potential. However, one can easily show that the path integral over the fluctuations is independent of the branch chosen. Expanding the action around the classical paths one is left with the fluctuation path integral (45). Using (47) and (58) it may be written as

$$
\begin{aligned}
f(\theta) & =\prod_{n=1}^{\infty}\left(\frac{1}{N} \sqrt{\frac{1}{8 \pi \theta}} \int_{-\infty}^{\infty} \mathrm{d} Y_{n}\right) \exp \left(-\left[\frac{1}{8 \theta} \sum_{n=2}^{\infty} \lambda_{n} Y_{n}^{2}+V\left(Q, Y_{1}\right)\right]\right) \\
& =\sqrt{\frac{\lambda_{1}}{4 \pi \sin (\theta)}} K(Q),
\end{aligned}
$$

where

$$
K(Q)=\sqrt{\frac{1}{8 \pi \theta}} \int_{-\infty}^{\infty} \mathrm{d} Y_{1} \exp \left(-V\left(Q, Y_{1}\right)\right)
$$

gives the contribution of the marginal mode $Y_{1}$. Now, with (36) and (64) the equilibrium density matrix in the vicinity of the critical point $\left(\lambda_{1 c}, r_{c}\right)$ reads

$$
\rho_{\theta}(z, r)=\frac{1}{Z} \sqrt{\frac{\lambda_{1}}{4 \pi \sin (\theta)}} K(Q) \exp \left(-S_{c l}(Q, z, r)\right) .
$$

Usually, explicit results for the density matrix can be calculated only numerically. After solving the cubic equation of motion (30) for given $\lambda_{1}$ and $r$ one has to insert $Q$ into the action (36) and the fluctuation potential (58) and determine the fluctuation integral (64). All steps involve rather simple numerics only and some results will be presented in section 5 . As we have seen in section 4.3 the full fluctuation potential is needed when the coefficient of the second order term $\Lambda_{1}(Q)$ is of order $\epsilon$ or smaller. For $\left|\Lambda_{1}(Q)\right|$ larger than order $\epsilon$ the Gaussian approximation for the fluctuation potential is appropriate. Moving away from the critical point $\left(\lambda_{c}, r_{c}\right)$, where $\Lambda_{1}(Q)=0$, we reach values of $\left|\Lambda_{1}(Q)\right|$ of order $\epsilon$ by varying $\lambda_{1}$ by $\Delta \lambda_{1}$ of order $\epsilon$ or by varying $Q$ by $\Delta Q$ of order $\epsilon^{1 / 6}$. The corresponding variation $\Delta r$ of the coordinate $r$ is then determined by the cubic equation (30). In the first case, varying $\lambda_{1}$ for fixed $Q=Q_{c}$, we get $\Delta r=\mathcal{O}\left(a_{3}\right)$. In the other case, varying $Q$ for fixed $\lambda_{1}=\lambda_{c}$, 


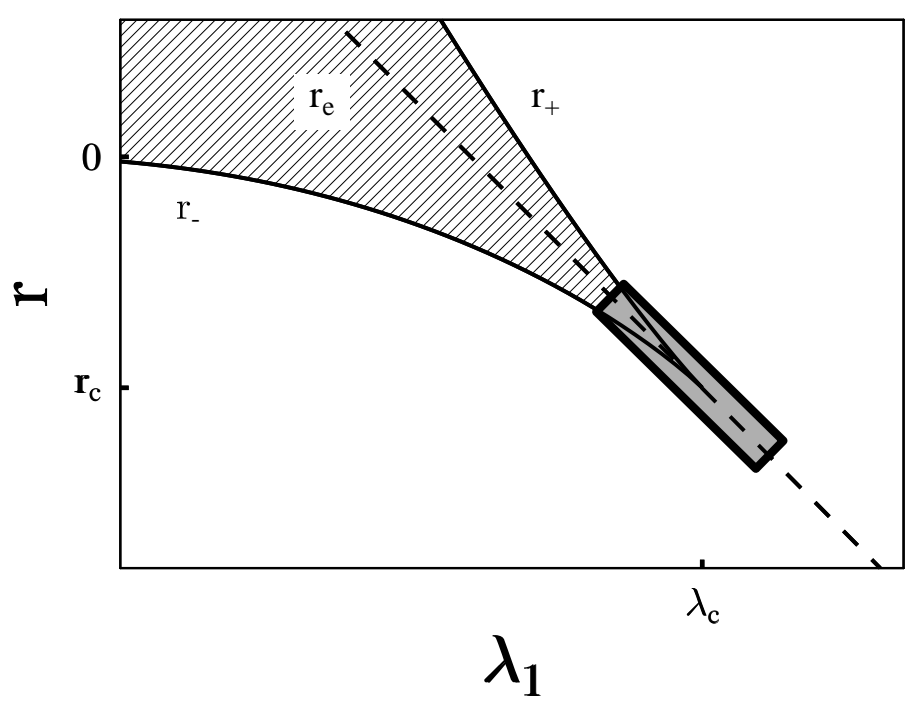

Fig. 4. Regions in $\lambda_{1}-r$-plane in which various results for the density matrix are valid. Around the critical point $\left(\lambda_{c}, r_{c}\right)$ in the dark region result (65) has to be used. In the white region, where only one solution of (30) exists, result (67) is appropriate, while in the shaded region result (70) holds. Along the dashed line $r_{e}\left(\lambda_{1}\right)$ introduced in (60) one has $Q=Q_{c}$

the order of magnitude of the variation of $r$ is $\Delta r=\mathcal{O}\left(\epsilon^{1 / 2}\right)$. Since $Q=Q_{c}$ along the line $r_{e}\left(\lambda_{1}\right)$ introduced in (60), the region around the critical point where the usual WKB approximation breaks down is of the form of the dark rectangle shown in Fig. 4. In this region the result (65) must be used.

For $\Lambda_{1}(Q)>\epsilon$ the fluctuation potential can be simplified to read

$$
V\left(Q, Y_{1}\right)=\frac{1}{8 \theta} \Lambda_{1}(Q) Y_{1}^{2}+o(1)
$$

where the terms omitted are smaller than order 1 since the fluctuations $Y_{1}$ are now smaller than $\epsilon^{-1 / 2} . K(Q)$ is therefore a Gaussian integral and the density matrix (65) reduces to

$$
\rho_{\theta}(z, r)=\frac{1}{Z} \sqrt{\frac{\lambda_{1}}{4 \pi \sin (\theta) \Lambda_{1}(Q)}} \exp \left(-S_{c l}(Q, z, r)\right) .
$$

For high temperatures, i.e. $\theta \ll \pi$, one sees from (40) and (55) that the result (67) matches into the density matrix in the high temperature limit (53).

On the other hand, as discussed in section 3.1, for temperatures below the critical region the cubic equation has two stable solutions which we call here $Q_{s 1}$ and $Q_{s 2}$ where $Q_{s 1} \leq Q_{s 2}$. As we have seen above, for $\Lambda_{1}(Q)<-\epsilon$ these 
two branches are well separated in function space, and a Gaussian approximation for the fluctuations around these stable paths is appropriate. Choosing $Q=Q_{s 1}$ the fluctuation potential has a minimum at $Y_{1}=0$ and it can be expanded to give

$$
V\left(Q_{s 1}, Y_{1}\right)=\frac{1}{8 \theta} \Lambda_{1}\left(Q_{s 1}\right) Y_{1}^{2}+o(1)
$$

while near the other minimum at $Y_{1}=Y_{+}-Y_{-}$the fluctuation potential takes the form

$$
\begin{aligned}
V\left(Q_{s 1}, Y_{1}\right) & =S_{c l}\left(Q_{s 2}, z, r\right)-S_{c l}\left(Q_{s 1}, z, r\right) \\
& +\frac{1}{8 \theta} \Lambda_{1}\left(Q_{s 2}\right)\left[Y_{1}-\left(Y_{+}-Y_{-}\right)\right]^{2}+o(1) .
\end{aligned}
$$

The density matrix therefore reads

$$
\begin{array}{r}
\rho_{\theta}(z, r)=\frac{1}{Z} \sqrt{\frac{\lambda_{1}}{4 \pi \sin (\theta)}}\left(\frac{1}{\sqrt{\Lambda_{1}\left(Q_{s 1}\right)}} \exp \left(-S_{c l}\left(Q_{s 1}, z, r\right)\right)\right. \\
\left.+\frac{1}{\sqrt{\Lambda_{1}\left(Q_{s 2}\right)}} \exp \left(-S_{c l}\left(Q_{s 2}, z, r\right)\right)\right) .
\end{array}
$$

This result matches onto (65) for values of $\lambda_{1}$ and $r$ where the coefficient $\Lambda_{1}(Q)$ becomes of order $\epsilon$ or smaller. When the difference of the actions of the two stable paths $\left|S_{c l}\left(Q_{s 1}, z, r\right)-S_{c l}\left(Q_{s 2}, z, r\right)\right|$ is of order 1 or larger, only the path with smaller action contributes to the path integral. The density matrix then reduces to

$$
\rho_{\theta}(z, r)=\frac{1}{Z} \sqrt{\frac{\lambda_{1}}{4 \pi \sin (\theta) \Lambda_{1}\left(Q_{s}\right)}} \exp \left(-S_{c l}\left(Q_{s}, z, r\right)\right),
$$

where $Q_{s}$ denotes the path with smaller action.

\section{Discussion and Conclusions}

We have studied the equilibrium density matrix of a quantum particle near the top of a general anharmonic potential barrier in the temperature range where large quantum fluctuations render the harmonic approximation of the 


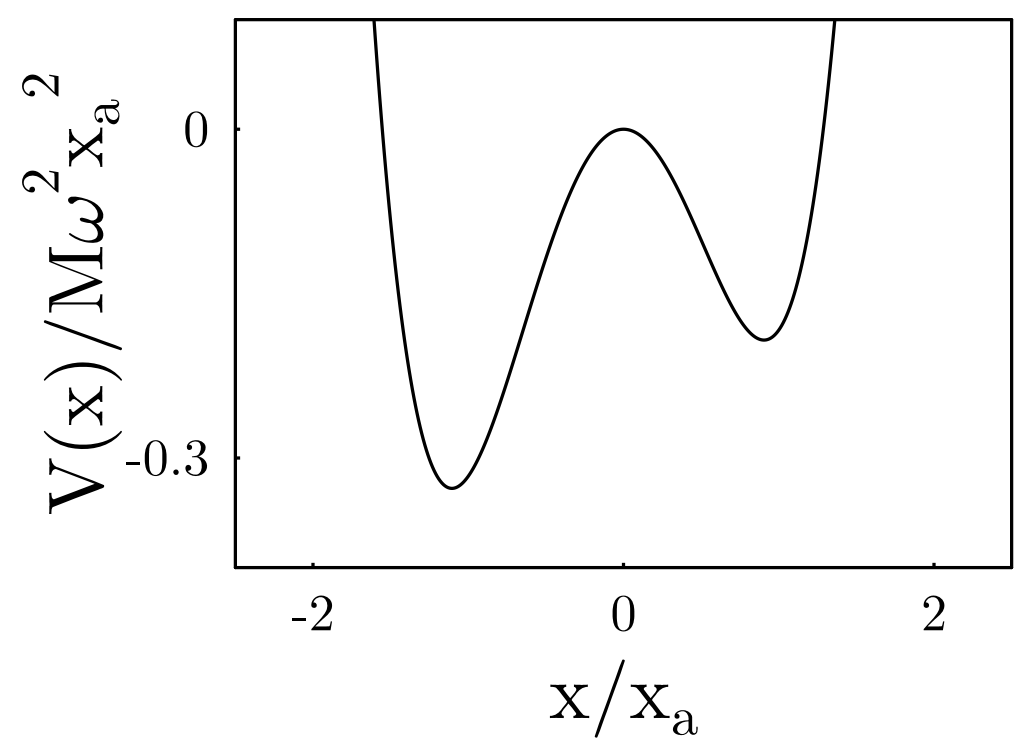

Fig. 5. The potential (8) with potential parameters $a_{3}=1 / 5, a_{4}=1$, and $a_{n}=0$ for $n \geq 5$.

potential insufficient even for coordinates in the vicinity of the barrier top. We have shown how the density matrix can be calculated by means of the path integral technique employing an expansion about classical paths. In a narrow range around the critical temperature $T_{c}$ and in the vicinity of the critical coordinate $r_{c}$ the Gaussian approximation for the quantum fluctuations, i.e. the simple WKB expansion, fails and one has to take into account higher order variational terms. The anharmonicity of the potential remains essential below the critical temperature.

To illustrate our results we have evaluated the diagonal part of the density matrix, i.e. the position distribution function $P(q)=\rho(z=0, r=q)$, for a system with the potential parameters $a_{3}=1 / 5$, and $a_{4}=1$ while all other coefficients $a_{n}=0$. Thus the potential (8) reduces to

$$
V(x)=-\frac{1}{2} M \omega^{2} x^{2}\left[1-\frac{2}{15} \frac{x}{x_{a}}-\frac{1}{2}\left(\frac{x}{x_{a}}\right)^{2}\right]
$$

describing an asymmetric double well (Fig. 5). Using the dimensionless formulation (12) the dimensionless potential $\bar{V}=V / \hbar \omega$ reads

$$
\bar{V}(q)=-\frac{1}{4} q^{2}+\frac{\epsilon}{30} q^{3}+\frac{\epsilon^{2}}{8} q^{4} .
$$

The explicit results given above hold for weakly asymmetric potentials with $a_{3}^{3} \leq \epsilon \ll 1$. This is the case for $\epsilon=0.01$. 


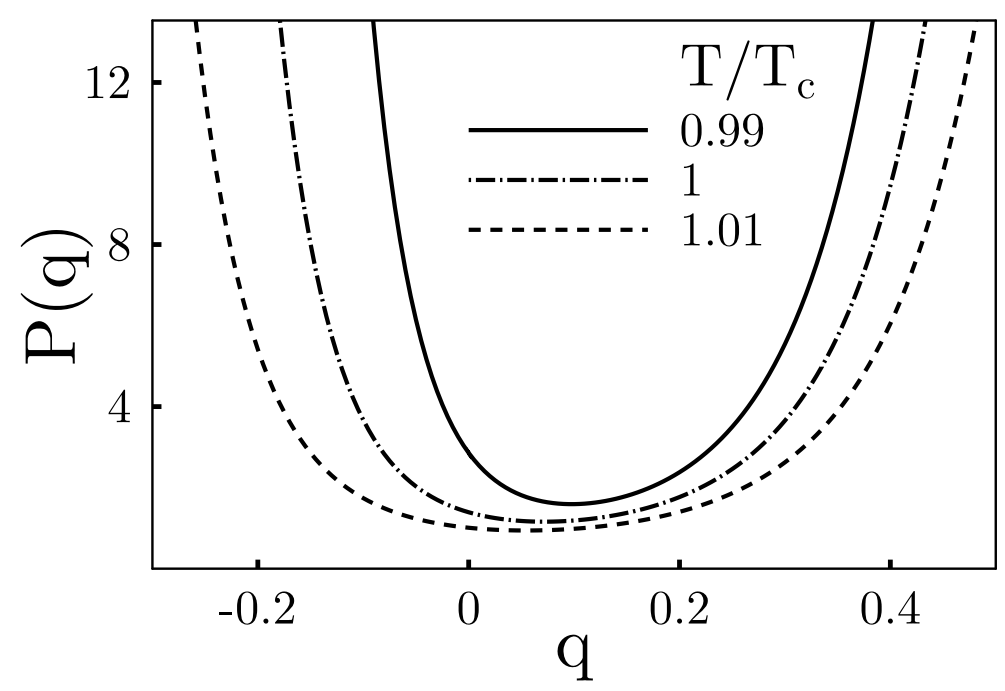

Fig. 6. The position distribution $P_{\theta}(q)$ at temperatures near $T_{c}$ as a function of $q$ near the barrier top.

In Fig. 6 the equilibrium position distribution function $P(q)$ is depicted for various temperatures near $T_{c}$. [The normalization factor for all figures shown in this section is choosen as $Z=1$.] For high temperatures the position distribution function is given by the harmonic approximation that is symmetric around the barrier top at $q=0$. Anharmonic terms in the equation of motion (30) become relevant with decreasing temperature. Accordingly, the minimum of the distribution is shifted and the distribution becomes more asymmetric as the temperature decreases.

In Fig. 7 we show the diagonal part of the density matrix as a function of the eigenvalue $\lambda_{1}$. At the barrier top, i.e. at $q=0$ (Fig. 7 $7 \mathrm{a}$ ) and for high temperatures there exist only one solution of (30), $Q=0$, and the distribution function is determined by (67). At $\lambda_{1}=\lambda_{0}$ a new stable solution $Q_{s}$ of (30) emerges. Between $\lambda_{1}=\lambda_{0}$ and $\lambda_{1}=0$ it exchanges global stability with the $Q=0$ solution and for lower temperatures the position distribution function is given by (71). While the results (67) and (71) diverge at $\lambda_{1}=0$ and $\lambda_{1}=$ $\lambda_{0}$, respectively, the general formular (65) matches between these results and remains finite.

For the critical value of the coordinate $q=r_{c}$ and at $\lambda_{1}=\lambda_{c}$ there exists only one solution $Q=Q_{c}$ of (30). The simple semiclassical approximation (67) diverges at $\lambda_{1}=\lambda_{c}$ and we have to take the general result (65). In Fig. 7 we see the matching of the result (65) with the result (67) valid below and above the critical region.

In summary, we have shown how to evaluate the semiclassical density matrix 

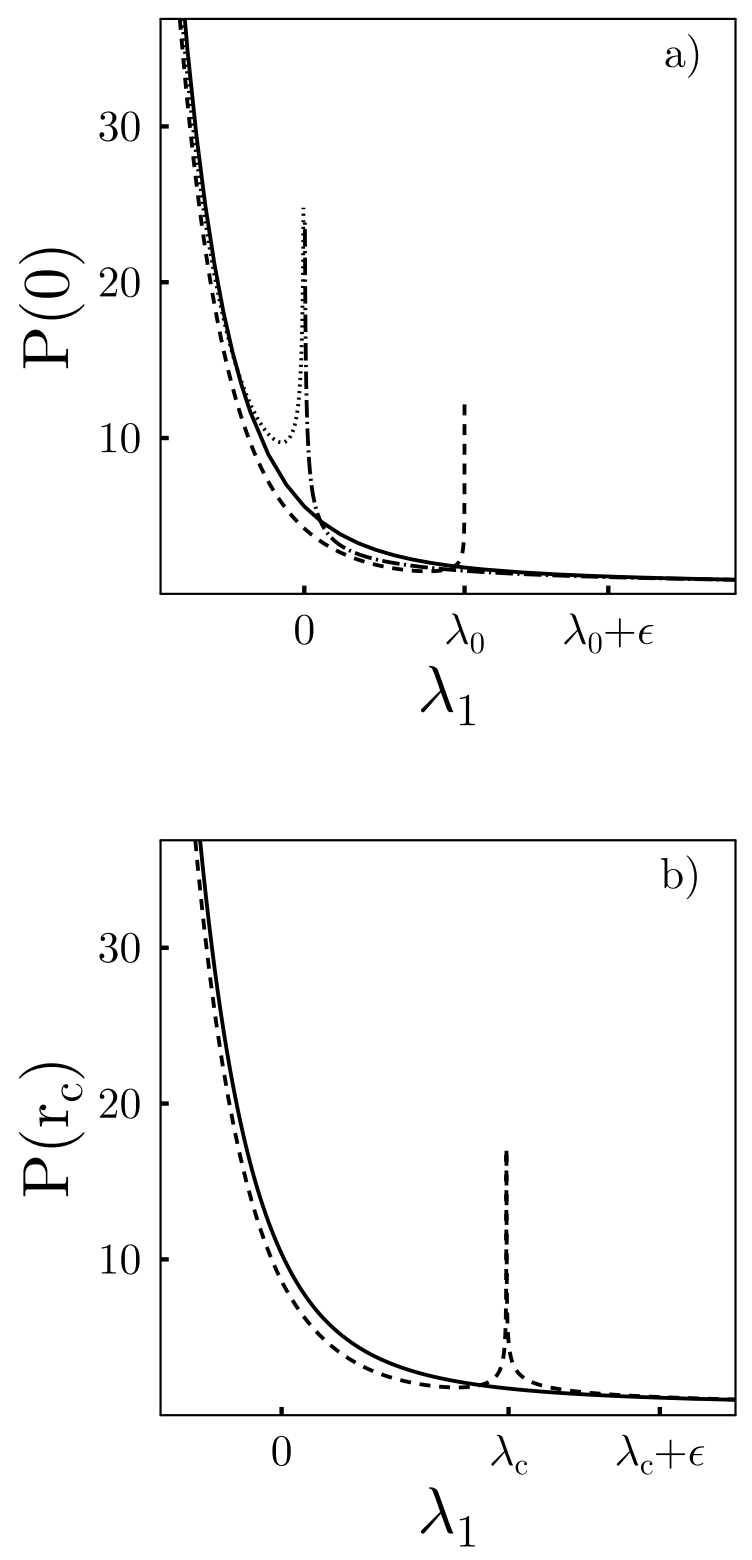

Fig. 7. The position distribution $P_{\theta}(q)$ at temperatures near $T_{c}$ as a function of the eigenvalue $\lambda_{1}$ for (a) $q=0$ and (b) $q=r_{c}$. The solid line represents the general result (65). Furthermore, various approximation are shown. In (a) the dashed line represents result (71) while the dotted line represents result (70). The dotted-dashed line represents result (67), which is for $q=0$ the same as the high temperature WKB approximation (53). In (b) the dashed lines represent result (67).

consistently near the critical region. For $a_{3}=0$ one regains the results for the anharmonic symmetric barrier potential investigated previously [6]. In this case the critical point $\left(\lambda_{c}, r_{c}\right)=(0,0)$. A comparison of the semiclassical density matrix with exact results will be given elsewhere. 


\section{References}

[1] P. Hänggi, P. Talkner, and M. Borkovec, Rev. Mod. Phys. 62 (1990) 251.

[2] R.P. Feynman and A.P. Hibbs, Quantum Mechanics and Path Integrals (McGraw-Hill, New York, 1965). R. P. Feynman, Statistical Mechanics (Benjamin, New York, 1972).

[3] L.S. Schulman, Techniques and Applications of Path Integrals (Wiley, New York, 1981).

[4] H. Kleinert, Path Integrals in Quantum Mechanics, Statistics and Polymer Physics (World Scientific, Singapore, 1990).

[5] H. Grabert, P. Olschowski, and U. Weiss, Phys. Rev. B 36 (1987) 1931

[6] J. Ankerhold, and H. Grabert, Physica A 188 (1992) 568.

[7] J. Ankerhold, F.-J. Weiper, and H. Grabert in: Inomata et al. (eds.), Path Integrals from meV to MeV (World Scientific, Singapore 1993).

[8] G. Iooss and D.D. Joseph, Elementary Stability and Bifurcation Theory (Springer, New York, 1989). 